\title{
Adjacent Channel Interference Modeling of Single Vibration Point on Multichannel Dynamic Pressure Sensors
}

\author{
Xingguang Geng $\mathbb{D}^{1,2,3}$ Su Liu, ${ }^{4}$ Yitao Zhang, ${ }^{1,3}$ Shaolong Zhang, ${ }^{1,3}$ Jiena Hou, ${ }^{1,2,3}$ \\ Jun Zhang, ${ }^{1,2,3}$ Muhammad Asif, ${ }^{1,5}$ and Hai-Ying Zhang ${ }^{1,2,3}$ \\ ${ }^{1}$ Institute of Microelectronics of Chinese Academy of Sciences, China \\ ${ }^{2}$ University of Chinese Academy of Sciences, China \\ ${ }^{3}$ Beijing Key Laboratory for Next Generation RF Communication Chip Technology, China \\ ${ }^{4}$ Navinfo Co., Ltd., China \\ ${ }^{5}$ Qurtuba University of Science and Information Technology KPK, Pakistan
}

Correspondence should be addressed to Hai-Ying Zhang; zhanghaiying@ime.ac.cn

Received 7 October 2019; Revised 28 November 2019; Accepted 26 December 2019; Published 12 February 2020

Academic Editor: Grigore Stamatescu

Copyright (c) 2020 Xingguang Geng et al. This is an open access article distributed under the Creative Commons Attribution License, which permits unrestricted use, distribution, and reproduction in any medium, provided the original work is properly cited.

\begin{abstract}
Pulse waves of a radial artery under different pressures applied through a cuff play an important role in disease diagnosis, especially in traditional chinese medicine (TCM). Pulse waves could be collected by a pressure sensor array affixed to an inflatable cuff. During a process of collecting pulse waves, one sensor of a sensor array moves up and down when the sensor is shocked by a pulse wave. Movement of the sensor leads to the passive displacement of other nearby sensors because of a connecting structure between them. Then, vibration signals will be generated by the nearby sensors although these sensors do not receive radial artery pulse waves. These vibration signals considered an interference are usually superimposed on real signals obtained from these nearby sensors and degrade signal quality. The problem mentioned above does not only generally exist in a pressure sensor array attached to a wristband but also is easy to ignore. This paper proposes a novel interference suppression algorithm based on Welch's method for estimating and weakening adjacent sensor channel interference to overcome the problem. At first, a sensor array attached to an inflatable cuff and a vibration generator is proposed to establish an experimental platform for simplifying the pulse wave collection process. Then, the interference suppression algorithm is proposed according to mechanical analysis and Welch's method based on the proposed sensor array and vibration generator. Next anti-interference abilities of the algorithm based on a simplified process are evaluated by different vibration frequencies and applied pressures. The anti-interference abilities of the algorithm based on pulse waves of the radial artery are evaluated indirectly. The results show that the novel interference suppression algorithm could weaken adjacent sensor channel interference and upgrade the signal quality.
\end{abstract}

\section{Introduction}

Radial pulse waves carry important physiological and pathological information and have been proven effective for predicting and reflecting diseases like hypertension, stroke, and diabetes mellitus [1-3]. The energy of the pulse wave is concentrated in $1-10 \mathrm{~Hz}[4]$. With the development of sensor technology, many new theories and new types of sensors are applied to detecting pulse waves including piezoresistive sensors [5-7], piezoelectric sensors [8-10], photoelectric sensors [11-13], and ultrasonic sensors $[14,15]$.
In TCM, an oriental medical doctor divides the terminal region of the wrist radial artery into three positions, called cun, guan, and chi, which can reflect physiological and pathological information of different viscera, respectively. To sense the changing trend of pulse waves, doctors apply static information of different pressure intensities to radial arteries of these three parts, called Fu, Zhong, and Chen, then judge the patient's condition [16]. Therefore, one pulse-taking detection system must provide both wrist artery pulse information and static hold-down pressure. In recent years, many studies focused on multitype sensor design and correlation 
analysis of cun, guan, and chi in TCM [17-21]. Based on these studies, many different pulse detection instruments were designed. They exerted variable static pressures on the three points of cun, guan, and chi and collected pulse waves using multitype sensors. However, there are adjacent interference signals among the multichannel pressure sensors. According to the traditional theory of pulse diagnosis in TCM, Wang et al. designed a multichannel pulse detection system based on a flexible array sensor [22]. A special pulse transducer is made of silica gel, and the pressure can be adjusted automatically by means of balloon pressurization. When a transducer displaces due to the vibration of the vessel, the balloon on the transducer will be deformed, and then the adjacent transducer will be forced to displace, resulting in interference signals. A novel multichannel wrist pulse system with different sensor arrays designed by Wang et al. [23], a Bi-Sensing Pulse Diagnosis Instrument (BSPDI) designed by Luo et al. [18], and a pulse-taking platform with a tactile array sensor designed by et al. [24] can collect pulse wave under different pressures by manual or mechanical pressure. The sensors are directly connected by a rigid structure. When a sensor displaces due to the vibration of the vessel, the rigid structure of the sensor will be displaced, which will change the pressure of the adjacent sensor and cause interference. The interference signal will be superimposed on the useful signal, which will affect the authenticity of the signal. The problem mentioned above does not only generally exist in a pressure sensor array attached to a wristband but also is easy to ignore. A novel flexible pressure sensor array for depth information of the radial artery designed by Liu et al. weakens the interference between sensors by adding sponges between sensors and airbags [25]. Few people have proposed an algorithmic solution.

Generally speaking, due to the direct or indirect physical links among sensors, the displacement of a sensor unit caused by vibration will interfere with the adjacent sensor by a connecting medium, and then affect the acquisition waveform of the adjacent sensor. However, the interference will affect the morphological characteristics of the pulse wave. To overcome this problem, one adjacent interference model of multichannel pressure sensors is proposed in this study. According to the structural characteristics of the multichannel pulse wave acquisition instrument, a simple mechanical structure model of the interference signal is established. Based on the mechanical structure model, the characteristics of interference signals (amplitude, frequency, and phase information) and interference signal expressions are determined. Welch's average cycle method is used to establish the transmission channel model. In this paper, the output signal of the dynamic pressure sensor on the vibration source is defined as channel input and the output signal of the proximity sensor is defined as channel output.

\section{Materials and Methods}

2.1. A Signal Acquisition Instrument. In this paper, a novel flexible pressure sensor array signal acquisition instrument is used to realize the signal collection [25]. This instrument is composed of three compound pressure sensors to realize the simultaneous measurement of three fluctuation points. As is showed in Figures 1(a) and 1(b), each compound pressure sensor consists of a piezoelectric sensor and a piezoresistive sensor, which are used to measure pulse wave and static pressure separately. A bending structure of the sensor could guarantee the signal intensity and prevent the occurrence of an inverted waveform. Figures 1(c) and 1(d) show that a polyurethane sponge is used to eliminate the propagation of contactor vibration effectively. An inflatable cuff on the wrist is used as a pressure device in the whole collecting process which could provide stepped pressures from 10 to $140 \mathrm{mmHg}$ in an interval of $10 \mathrm{mmHg}$. In each pressure step, the cuff is inflated to produce a specified pressure and lasts for several seconds. The pulse wave signals and static pressure information of three sensors are recorded in real-time until the next instruction of inflation comes. In this way, the pulse waves under a pressure gradient are acquired. After all the pressure steps finish, the cuff runs a deflation process and the whole collecting process is completed. The sampling frequency is approximately $225 \mathrm{~Hz}$ and the recording time is approximately $10 \mathrm{~s}$ in each pressure interval. To eliminate the propagation of contactor vibration effectively, a polyurethane sponge is used in the novel flexible pressure sensor array signal acquisition instrument. For the convenience of expression, the sensor group is named sensor $\mathrm{A}$, sensor $\mathrm{B}$, and sensor C, in turn.

2.1.1. Multipoint Vibration Source Generator. In order to study the influence of single or multiple vibration sources on multichannel sensor signals, a multivibrator signal generator is designed in this paper. As shown in Figure 2, the device roughly consists of a motor, a cam, a link mechanism, and a long contractor and three vibrate points. When the motor begins to run, the link mechanism is driven through the cam. Then, the long contactor could periodically move up and down to drive three vibrate points up and down and touch the surface of a sensor lightly. In order to study the interference of a single-point vibration source and mutual interference between multipoint vibration sources, three vibrate points can be disassembled and the height of three vibrate points can be adjusted. ADC motor controller is used to control vibration frequency. Increasing pressure is exerted on the sensor through an inflatable cuff. The dynamic pressures under variable static pressures are measured and recorded. The contraction of the human heart produces waves, which pass through the artery to the wrist radial artery to produce pulse waves. Pulse wave passes through three parts: chi, guan, and cun. This means that the vibration time of the three parts is inconsistent. However, the average conduction velocity of the human pulse wave is $15 \mathrm{~m} / \mathrm{s}$. The maximum distance from chi to guan is $34 \mathrm{~mm}$, and the signal sampling frequency is 225 . The time required for a pulse wave to pass from chi to cun is $0.002 \mathrm{~s}$, which is less than the sampling interval of data $0.0044 \mathrm{~s}$. Therefore, the lag of three parts cannot be considered when making a signal generator.

2.2. Dynamic Mechanical Analysis. When the piezoelectric material is pressed, it generates polarization charges, and its signal amplitude and frequency are directly proportional to 


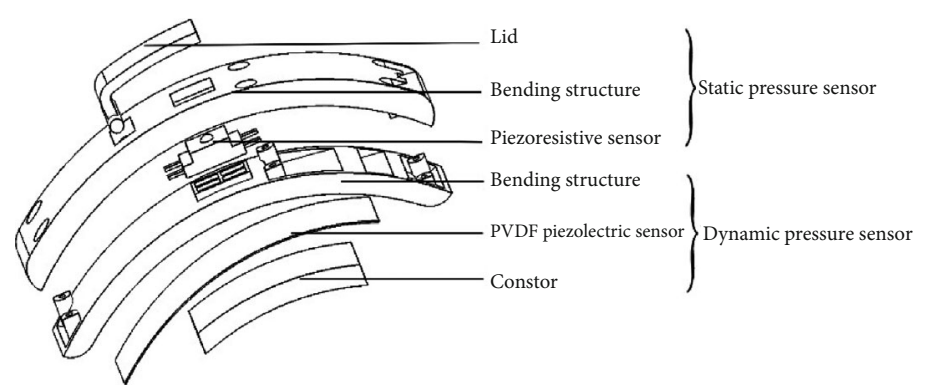

(a)

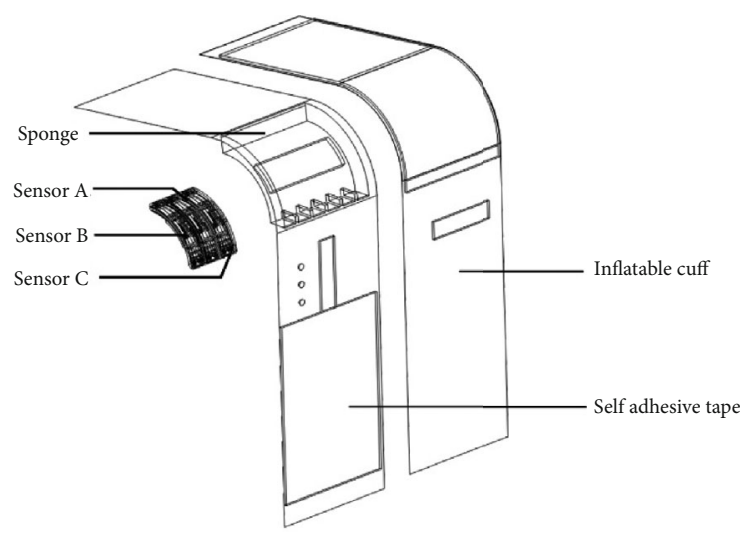

(c)

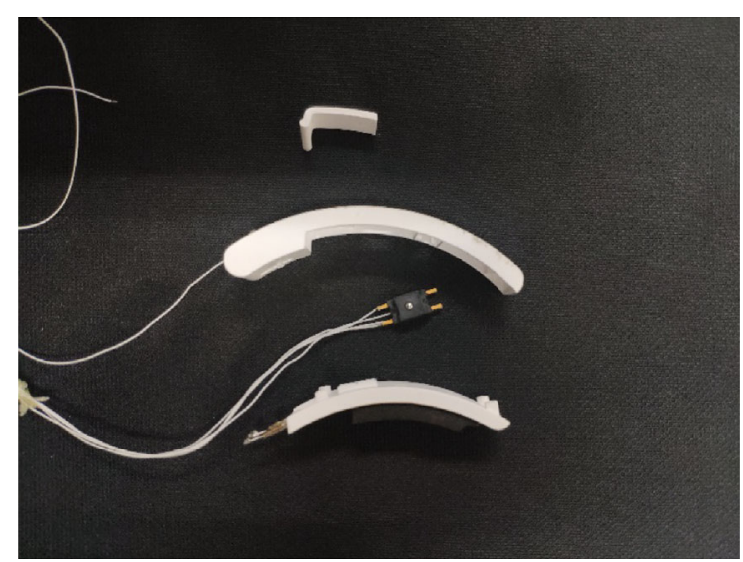

(b)

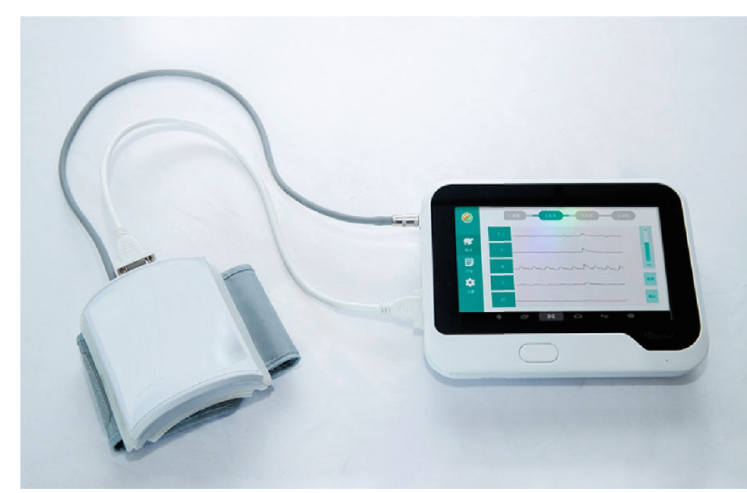

(d)

Figure 1: (a) Schematic diagram of the compound pressure sensor. (b) Photograph of the compound pressure sensor. (c) Photograph of the wristband structure. (d) Schematic diagram of the wristband.

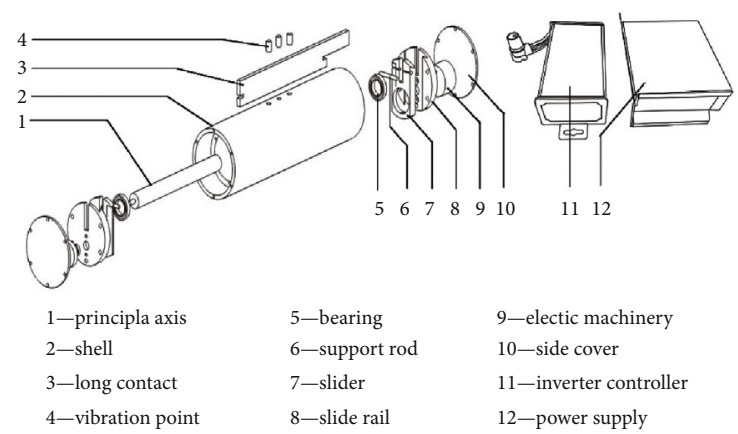

(a)

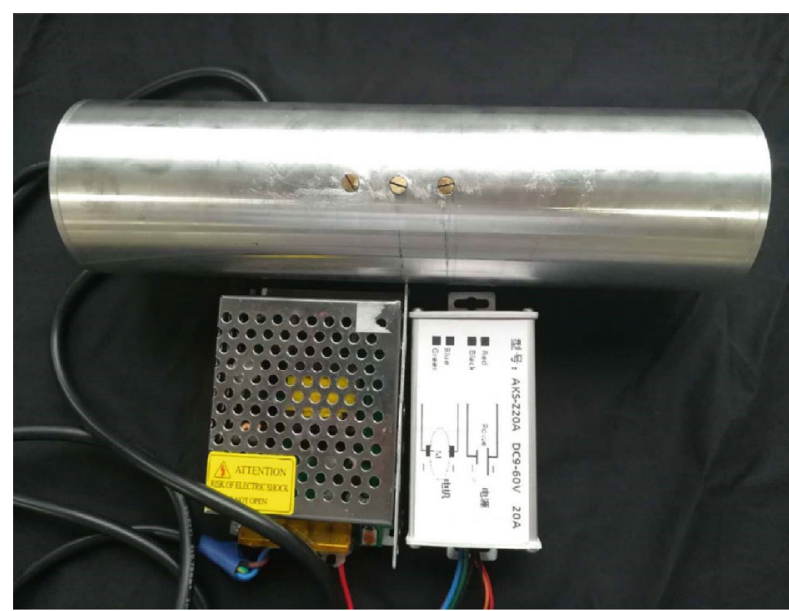

(b)

Figure 2: (a) Schematic diagram of the multipoint vibration source generator. (b) Photograph of the multipoint vibration source generator.

the mechanical deformation of the piezoelectric material. Deformation changes the charge density on the surface of the material, thus generating a voltage between the surfaces with electrodes. When the applied force is reversed, the polarity of the output voltage is also reversed. A reciprocating force gives an alternating output voltage. For the dynamic 


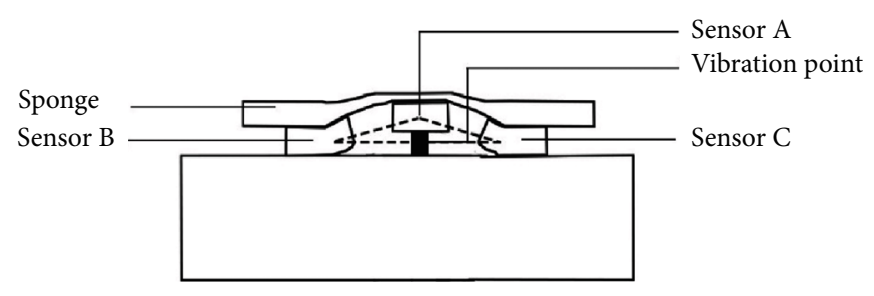

Figure 3: Profile of the sponge deformation under the force.

pressure sensor, the magnitude of the output signal of the sensor is related to the shape variable of the piezoelectric sensor. As the shape variable increase, then the amplitude of the output signal of this sensor is increased. When the shape variable is reduced, then the amplitude of the output signal of the piezoelectric sensor is reduced. To simplify the complexity of the mechanical structure model, this paper just only analyzes the effects of the single-point vibration source on the proximity sensor.

As is showed in Figure 3, when the airbag in the inflatable cuff is pressurized to a constant value, the dynamic pressure sensors in the compound pressure sensors are deformed to one constant under the action of air pressure. When the vibration source moves upwards, sensor A is driven upward by the upward support force of the vibration point and the shape variable of sensor $A$ is increased. The sponge around sensor A is compressed by force.

When the vibration source moves upward, the pressure on sensor B film decreases gradually and the deformation decreases, and then the output signal amplitude of sensor B decreases gradually. When the output signal amplitude of sensor A increases to the maximum, the output signal amplitude of sensor B decreases to the minimum.

When the vibration source moves downward, the strain pressure of sensor A film decreases. As the sponge depression height of sensor A decreases, the signal amplitude of sensor A decreases gradually. When the output signal amplitude of sensor A decreases to the minimum, the output signal amplitude of sensor B increases to the maximum.

As previously described, compared with the output of the sensor A, the phase delay of the output signal of sensor B is 180 degrees. According to the attenuation characteristics of signal during transmission, the amplitude of the output signal of sensor B is smaller than that received by the dynamic pressure sensor $\mathrm{A}$.

It is assumed that the output signal of sensor $\mathrm{A}$ is a sinusoidal function. It is defined as follows:

$$
f(x)=\sin (1 \pi f x) .
$$

In equation (1), $f$ is the signal frequency. The expression of the output signal of sensor B can be defined as follows:

$$
g(x)=\delta \sin (2 \pi f x+\pi)
$$

According to the two equations above, we just need to determine the attenuation coefficient; then, we can get the expression of the interference signal.
2.3. Transmission Channel Model. In formula (2), $\delta$ is related to the attenuation of the channel between the two sensors. The signal transmission function reflects the transmission characteristics of the channel. Welch's average cycle method is the most commonly used method to calculate the transmission function of one system. If the input and output signals are known, the transmission function of the system is estimated by the Welch's average cycle method.

$$
\begin{aligned}
P_{x x}(\omega) & =\sum_{m=-\infty}^{\infty} R_{x x}(m) e^{-j \omega m}, \\
P_{x y}(\omega) & =\frac{1}{2 \pi} \sum_{m=-\infty}^{\infty} R_{x y}(\omega) e^{-j \omega m}, \\
H(w) & =\operatorname{Pxy}(w) / \operatorname{Pxx}(w) .
\end{aligned}
$$

In equation (3), $P_{x x}(w)$ is the self-power spectral density (SPSD) of the input signal. $P_{x y}(w)$ is the cross power spectral density(CPSD) between input and output signals. In this paper, the output signal of sensor $A$ is defined as channel input and the output signal of sensor B is defined as channel output. To simplify the complexity of the interference signal model, this paper just only analyzes the effect of the singlepoint vibration source on sensor $B$. The single-point vibration signal is generated by a signal generator, and the vibration signal and interference signal under different pressures are collected by the signal acquisition instrument. For the signal acquisition instrument, the middle compound pressure sensor (A) collects the vibration signal, and the other two sensors (B and C) collect the interference signals.

Considering that the vibration frequency of the signal generator is limited, according to the results of mechanical analysis and input signal, the expression of the interference signal can be obtained. Assuming that the length of the input data is $N$, the number of points in the frequency domain within the range of $f_{0}$ is $M$, and the sampling frequency of the data is $f_{s}\left(f_{s}=225\right.$, in this paper $)$, the following expressions can be obtained:

$$
\begin{aligned}
\frac{w_{0}}{2 \pi} & =\frac{f_{s}}{N} M, \\
M & =\left\lfloor\frac{w_{0} N}{2 \pi f_{s}}\right\rfloor .
\end{aligned}
$$

In formula (4), $\lfloor\cdot\rfloor$ is the sign of downward integration. Assuming that in the adjacent sensor, the output signal 
is $y(n)$, then the expression of the signal in the digital domain of $0-f_{0}$ can be expressed as follows.

According to the discrete Fourier transform,

$$
X(k)=\sum_{n=0}^{N-1} x(n) e^{-j(2 \pi / N) n k}=\sum_{n=0}^{N-1} x(n) e^{-j w n k}, \quad w \in(0,2 \pi)
$$

According to the relationship between the input signal and output signal, the expression in this paper is as follows:

$$
Y(k)= \begin{cases}X(0), & k=0, \\ -\delta X(k), & k=1,2, \cdots M, \\ 0, & k=M+1, M+2, \cdots N-M-1, \\ -\delta X(k), & k=N-M, N-M+1, \cdots N-1 .\end{cases}
$$

According to the inverse Fourier transform, we can get the time domain expression of the output signal.

$$
\begin{aligned}
y(n)= & \frac{1}{N} \sum_{n=0}^{N-1} Y(k) e^{j k w_{0} n} \\
= & \frac{1}{N}\left[\sum_{k=1}^{M}-\delta X(k) e^{j k w_{0} n}+\sum_{k=M+1}^{N-M-1} X(k) e^{j k \omega_{0} n}\right. \\
& \left.+\sum_{k=N-M}^{N-1}-\delta X(k) e^{j k w_{0} n}+X(0)\right] .
\end{aligned}
$$

$X(01) / N$ in formula (7) is the DC component. According to the above expression, it can be realized by using MATLAB.

\section{Experimental Results}

According to the transmission function, amplitudefrequency characteristic (AFC) curve and phase frequency characteristic (PFC) curve can be obtained.

Figures 4(a) and 4(b) are the AFC curve and PFC curve between sensor $\mathrm{A}$ and sensor $\mathrm{B}$ and the $\mathrm{AFC}$ curve and PFC curve between sensor $A$ and sensor $C$ under the same airbag pressure, respectively. As shown in Figures 4(a) and $4(\mathrm{~b})$, in the range of $0 \sim 35 \mathrm{~Hz}$, the channel gain between sensor A and sensor B and the channel gain between sensor A and sensor $\mathrm{C}$ are constants and the constant values are equal. Meanwhile, in the range of $0-35 \mathrm{~Hz}$, the phase difference between sensor $\mathrm{A}$ and sensor $\mathrm{B}$ and the phase difference between sensor $\mathrm{A}$ and sensor $\mathrm{C}$ are 180 degrees. Within the effective frequency range, these indicate that the amplitude of the output signal of sensor B or sensor C is smaller than that received by sensor $\mathrm{A}$, and compared with the output of the dynamic pressure sensor above the vibration source, the phase delay of the output signal of sensor B or sensor C is 180 degrees.
Figures 4(c) and 4(d) are the AFC curves between sensor $\mathrm{A}$ and sensor $\mathrm{B}$ at $70 \mathrm{mmHg}$ and $100 \mathrm{mmHg}$ pressures. As shown in Figures $4(\mathrm{c})$ and $4(\mathrm{~d})$, in the range of $0 \sim 35 \mathrm{~Hz}$, the channel gain between sensor $\mathrm{A}$ and sensor $\mathrm{B}$ and the channel gain at $70 \mathrm{mmHg}$ and $100 \mathrm{mmHg}$ are constants and the constant values are equal. Meanwhile, in the range of $0-35 \mathrm{~Hz}$, the phase difference between sensor $\mathrm{A}$ and sensor $\mathrm{B}$ at $70 \mathrm{mmHg}$ and $100 \mathrm{mmHg}$ is 180 degrees. These indicate that at $70 \mathrm{mmHg}$ and $100 \mathrm{mmHg}$ sensors have the same effective frequency range and the channel gain and the phase difference are equal.

In order to verify the accuracy of the model, signal attenuation $(\mathrm{dB})$ is used as evaluation indicators, in which signal attenuation reflects the ability of the model to suppress interference signals. Figure 5(a) is an error bar graph of AFC between the input signal and the output signal at different pressures, while sensor A collects the input signal and sensor $\mathrm{B}$ and $\mathrm{C}$ collect the output signal. The abscissa axis of the figure is the air pressure value (unit: $\mathrm{mmHg}$ ), and the ordinate axis is the signal attenuation (unit: $\mathrm{dB}$ ). The number of measurements was 5 times. In the 5 measurements, the maximum signal attenuation is $22.6 \mathrm{~dB}$ and the minimum signal attenuation is $19.3 \mathrm{~dB}$. The maximum fluctuation range of signal attenuation is $+0.49 \mathrm{~dB}$, and the minimum fluctuation range of signal attenuation is $+0.06 \mathrm{~dB}$. This shows that the output signal of the multiple measurement models of the same object under test is stable. With the increase of static pressure, the attenuation of the $d_{0}$ remains unchanged, which indicates that the value of $\delta$ is the same under different pressures. When the horizontal distance between the two sensors is constant, the value of $\delta$ is basically constant.

Figure 5(b) is an error bar graph of AFC between the input signal and the output error signal at different pressures, while sensor A collects the input signal and the output error signal is the error value of the output signal. In the five measurements, the maximum signal attenuation is $45.9 \mathrm{~dB}$ and the minimum signal attenuation is $45.2 \mathrm{~dB}$. The maximum fluctuation range of signal attenuation is $\pm 1.6 \mathrm{~dB}$, and the minimum fluctuation range of signal attenuation is $\pm 0.3 \mathrm{~dB}$. Compared with Figure 5(a), Figure 5(b) shows that the model can attenuate the output interference signal more than $30 \mathrm{~dB}$.

\section{Discussion and Conclusions}

In this paper, the mechanical analysis of composite sensors is presented. It is showed that the phase difference between the signal collected by the composite sensor and the crosstalk signal is 180 degrees, and the amplitude ratio of the signal is one attenuation factor $\delta$. The self-made signal generator is used to analyze the transmission characteristics of the interference signal under a single-point vibration source. The effective frequency range of the vibration source signal produced by the signal generator is $0 \sim 35 \mathrm{~Hz}$. Within this frequency range, the phase difference between the signal collected by the sensor and the crosstalk signal is 180 degrees. The output model of the interference signal is established by using the Fourier transform. The experimental result proves that this signal model can well construct the output model of the interference signal. The model can attenuate 


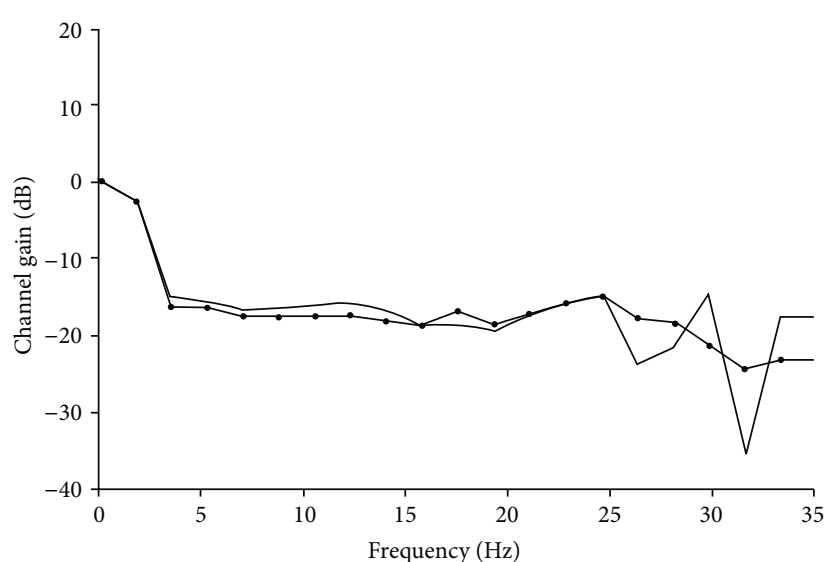

- AFC curve between A and B

$\rightarrow$ AFC curve between A and C

(a)

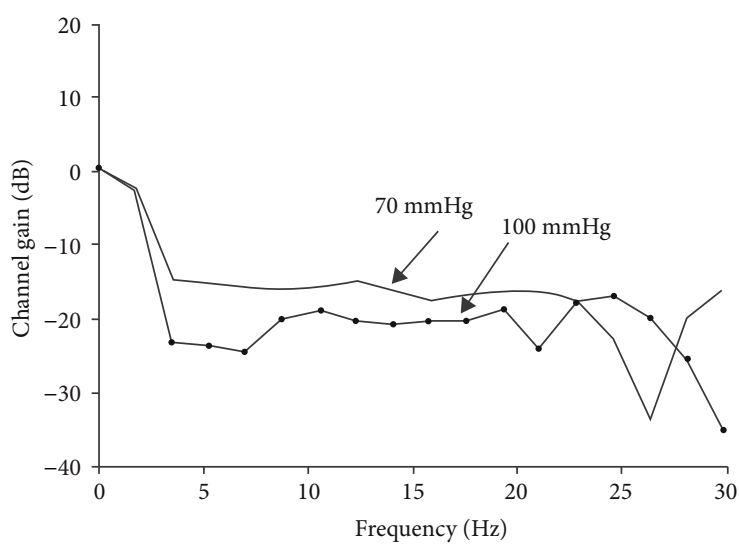

(c)

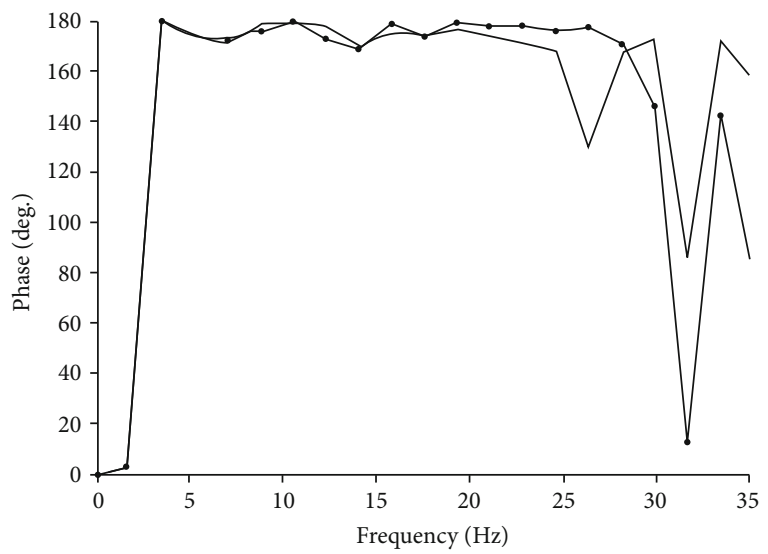

- PFC curve between sensor A and B

$\rightarrow$ PFC curve between sensor A and C

(b)

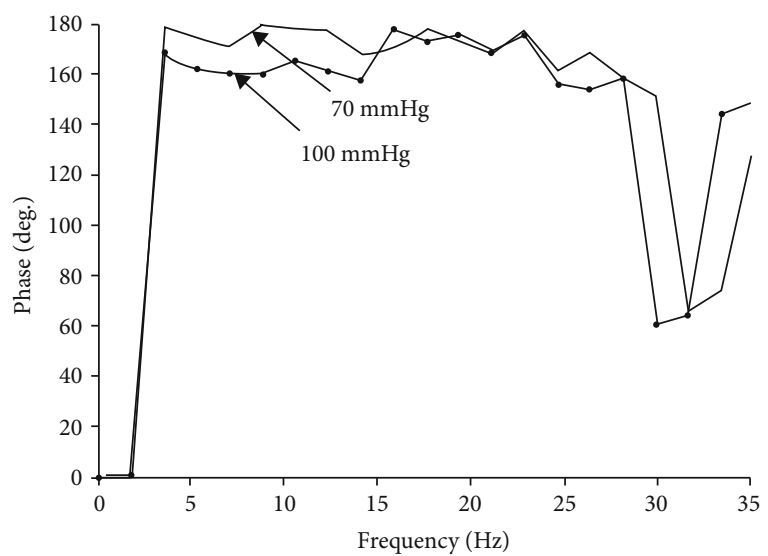

(d)

FIGURE 4: (a) AFC curve under the same airbag pressure. (b) PFC curve under the same airbag pressure. (c) AFC curve under the different airbag pressures. (d) PFC curve under different airbag pressures.

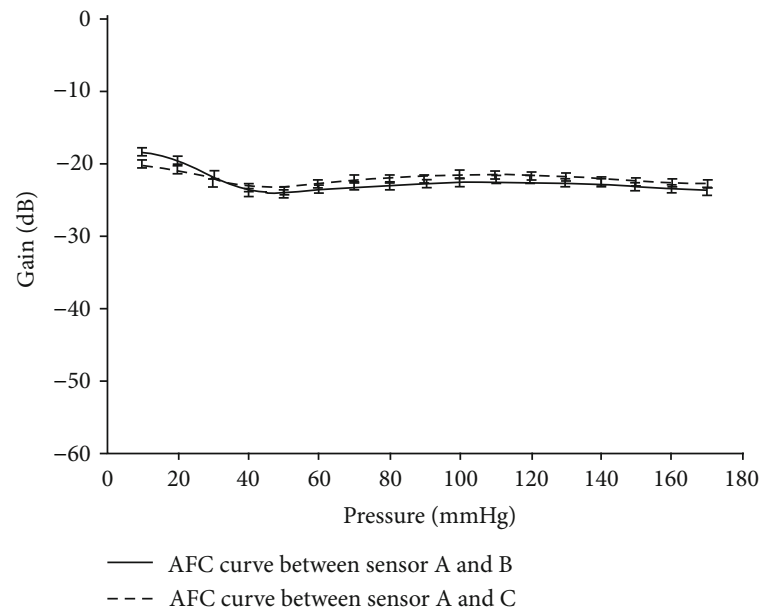

(a)

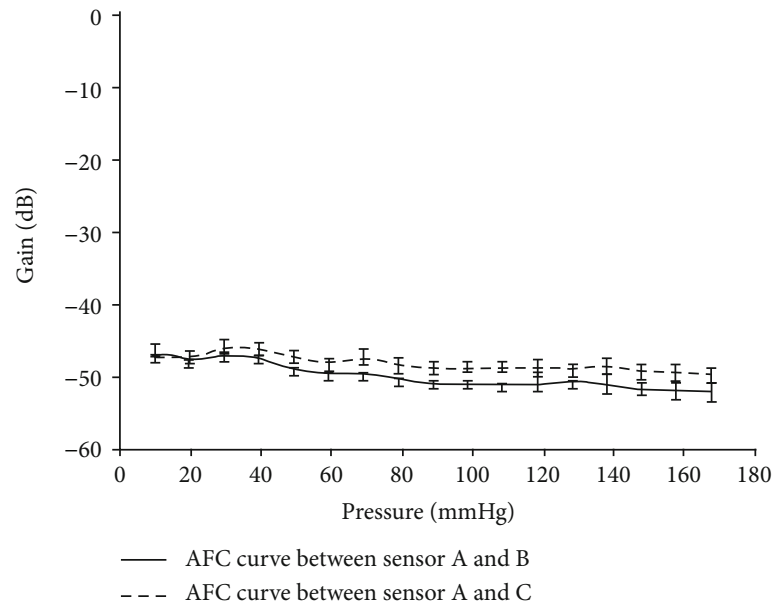

(b)

Figure 5: (a) AFC curves between the input signal and the output signal at different pressures. (b) AFC curves between the input signal and the output error signal at different pressures. 
the interference signal by $30 \mathrm{~dB}$ and has good repeatability. Meanwhile, the magnitude of the crosstalk signals of A-B and $\mathrm{A}-\mathrm{C}$ are equal, which indicates that the same vibration source has the same interference intensity to the sensors with the same distance on both sides. The results can reduce the complexity of the multichannel interference signal model. This technology is a common technology for interference suppression of a multisensor array and can be used in the field of interference suppression of array antenna reception.

\section{Data Availability}

The raw/processed data required to reproduce these findings cannot be shared at this time as the data also forms part of an ongoing study.

\section{Additional Points}

The works presented in this paper are subject to pending China and international patents filed by the Institute of Microelectronics of the Chinese Academy of Sciences (IMECAS) in China (PCT/CN2017/092400, PCT/CN2017/092388, and PCT/CN2017/092399).

\section{Conflicts of Interest}

The authors declare that there is no conflict of interests regarding the publication of this article.

\section{Acknowledgments}

This study was supported by funds from the National Science and Technology Major Project of China (No. 2018ZX01031-201) and Key Deployment Projects of Chinese Academy of Science (No. KFZD-SW-218). This work was supported by grants from the National Key Research and Development Program of China (2018YFC2001200). The authors thank the reviewers for the valuable feedback of the paper.

\section{References}

[1] M. Koizumi, H. Shimizu, K. Shimomura et al., "Relationship between hyperinsulinemia and pulse wave velocity in moderately hyperglycemic patients," Diabetes Research and Clinical Practice, vol. 62, no. 1, pp. 17-21, 2003.

[2] T. Takami and M. Shigemasa, "Efficacy of various antihypertensive agents as evaluated by indices of vascular stiffness in elderly hypertensive patients," Hypertension Research, vol. 26, no. 8, pp. 609-614, 2003.

[3] F. U. S. Mattace-Raso, T. J. M. van der Cammen, A. Hofman et al., "Arterial stiffness and risk of coronary heart disease and stroke: the Rotterdam Study," Circulation, vol. 5, pp. 657-663, 2006.

[4] C. M. Huang, C. C. Wei, Y. T. Liao, H. C. Chang, S. T. Kao, and T. C. Li, "Developing the effective method of spectral harmonic energy ratio to analyze the arterial pulse spectrum," Evidence-based Complementary and Alternative Medicine, vol. 2011, Article ID 342462, 7 pages, 2011.

[5] Y. Chen, B. Lu, Y. Chen, and X. Feng, "Biocompatible and ultra-flexible inorganic strain sensors attached to skin for long-term vital signs monitoring," IEEE Electron Device Letters, vol. 37, no. 4, pp. 496-499, 2016.

[6] P. Wang, W. Zuo, and D. Zhang, "A compound pressure signal acquisition system for multichannel wrist pulse signal analysis," IEEE Transactions on Instrumentation and Measurement, vol. 6, pp. 1556-1565, 2014.

[7] Z. Wang, S. Wang, J. Zeng et al., "High sensitivity, wearable, piezoresistive pressure sensors based on irregular microhump structures and its applications in body motion sensing," Small, vol. 12, no. 28, pp. 3827-3836, 2016.

[8] J. C. Murphy, K. Morrison, J. McLaughlin, G. Manoharan, and A. A. J. Adgey, "An innovative piezoelectric-based method for measuring pulse wave velocity in patients with hypertension," The Journal of Clinical Hypertension, vol. 13, pp. 497-505, 2011.

[9] J. McLaughlin, M. McNeill, B. Braun, and P. D. McCormack, "Piezoelectric sensor determination of arterial pulse wave velocity," Physiological Measurement, vol. 24, no. 3, pp. 693702, 2003.

[10] F. Clemente, P. Arpaia, and P. Cimmino, "A piezo-film-based measurement system for global haemodynamic assessment," Physiological Measurement, vol. 31, no. 5, pp. 697-714, 2010.

[11] Y. Sun, J.-J. Shuai, Q.-Y. Li, and J.-W. Yang, "Photoelectric sphygmograph sensor," Physics Experimentation, vol. 26, no. 5, pp. 13-17, 2006.

[12] L. S. Lovinsky, "Urgent problems of metrological assurance of optical pulse oximetry," IEEE Transactions on Instrumentation and Measurement, vol. 55, no. 3, pp. 869-875, 2006.

[13] S. Loukogeorgakis, R. Dawson, N. Phillips, C. N. Martyn, and S. E. Greenwald, "Validation of a device to measure arterial pulse wave velocity by a photoplethysmographic method," Physiological Measurement, vol. 23, no. 3, pp. 581-596, 2002.

[14] C. Huang, T.-L. Ren, and J. Luo, "Effects of parameters on the accuracy and precision of ultrasound-based local pulse wave velocity measurement: a simulation study," IEEE Transactions on Ultrasonics, Ferroelectrics, and Frequency Control, vol. 61, no. 12, pp. 2001-2018, 2014.

[15] M. Couade, M. Pernot, C. Prada et al., "Quantitative assessment of arterial wall biomechanical properties using shear wave imaging," Ultrasound in Medicine \& Biology, vol. 36, no. 10 , pp. 1662-1676, 2010.

[16] C.-H. Luo and C.-Y. Chung, "Non-invasive holistic health measurements using pulse diagnosis: II. Exploring TCM clinical holistic diagnosis using an ingestion test," European Journal of Integrative Medicine, vol. 8, no. 6, pp. 926-931, 2016.

[17] E. King, D. M. Cobbin, and S. Walsh, "The reliable measurement of radial pulse characteristics," Acupucture in Medicine, vol. 20, no. 4, pp. 150-159, 2002.

[18] C.-H. Luo, Y.-F. Chung, C.-S. Hu et al., "Possibility of quantifying TCM finger-reading sensations: I. Bi-sensing pulse diagnosis Instrument," European Journal of Integrative Medicine, vol. 4, no. 3, pp. e255-e262, 2012.

[19] Y.-F. Chung, C.-S. Hu, C.-H. Luo et al., "Possibility of quantifying TCM finger-reading sensations: II. An example of health standardization," European Journal of Integrative Medicine, vol. 4, no. 3, pp. e263-e270, 2012.

[20] Y.-W. Chu, C.-H. Luo, Y.-F. Chung, C.-S. Hu, and C.-C. Yeh, "Using an array sensor to determine differences in pulse diagnosis-three positions and nine indicators," European Journal of Integrative Medicine., vol. 6, no. 5, pp. 516-523, 2014. 
[21] U. Jaeuk, Y. J. L. Kim, J. Lee, and J. Y. Kim, "Differences in the properties of the radial artery between cun, guan, chi, and nearby segments using ultrasonographic imaging: a pilot study on arterial depth, diameter, and blood flow," Evidence-Based Complementary and Alternative Medicine, vol. 2015, Article ID 381634, 7 pages, 2015.

[22] X. M. Wang, C. Yang, X. Z. Lu, M. Luo, and P. Zhou, "The design of multi-channel pulse detection system based on flexible array sensor," Chinese Journal of Sensors and Actuators, vol. 25, 2012.

[23] D. Wang, D. Zhang, and G. Lu, "A novel multichannel wrist pulse system with different sensor arrays," IEEE Transactions on Instrumentation and Measurement, vol. 64, no. 7, pp. 2020-2034, 2015.

[24] C.-S. Hu, Y.-F. Chung, C.-C. Yeh, and C.-H. Luo, "Temporal and spatial properties of arterial pulsation measurement using pressure sensor array," Evidence-Based Complementary and Alternative Medicine, vol. 2012, Article ID 745127, 9 pages, 2012.

[25] S. Liu, S. Zhang, Y. Zhang, X. Geng, J. Zhang, and H. Zhang, "A novel flexible pressure sensor array for depth information of radial artery," Sensors and Actuators A: Physical, vol. 272, pp. 92-101, 2018. 